\title{
Reflection and transmission coefficients of a thin bed
}

\author{
Chun Yang ${ }^{1}$, Yun Wang ${ }^{1}$, and Yanghua Wang ${ }^{2}$
}

\begin{abstract}
The study of thin-bed seismic response is an important part in lithologic and methane reservoir modeling, critical for predicting their physical attributes and/or elastic parameters. The complex propagator matrix for the exact reflections and transmissions of thin beds limits their application in thin-bed inversion. Therefore, approximation formulas with a high accuracy and a relatively simple form are needed for thin-bed seismic analysis and inversion. We have derived thin-bed reflection and transmission coefficients, defined in terms of displacements, and approximated them to be in a quasi-Zoeppritz matrix form under the assumption that the middle layer has a very thin thickness. We have verified the approximation accuracy
\end{abstract}

through numerical calculation and concluded that the errors in PP-wave reflection coefficients $R_{\mathrm{PP}}$ are generally smaller than $10 \%$ when the thin-bed thicknesses are smaller than one-eighth of the PP-wavelength. The PS-wave reflection coefficients $R_{\mathrm{PS}}$ have lower approximation accuracy than $R_{\mathrm{PP}}$ for the same ratios of thicknesses to their respective wavelengths, and the $R_{\mathrm{PS}}$ approximation is not acceptable for incident angles approaching the critical angles (when they exist) except in the case of extremely strong impedance difference. Errors in phase for the $R_{\mathrm{PP}}$ and $R_{\mathrm{PS}}$ approximation are less than $10 \%$ for the cases of thicknesses less than one-tenth of the wavelengths. As expected, a thinner middle layer and a weaker impedance difference would result in higher approximation accuracy.

\section{INTRODUCTION}

As exploration targets have been expanded in scope from structural traps to lithologic and stratigraphic traps, more attention has been paid to thin-bed reservoirs that require higher resolution to be recognized (Zhang and Zheng, 2007). However, mature industrial amplitude variation with offset (AVO) inversion methods, which are based on the Zoeppritz equations and their approximation formulas, for describing reflection and transmission of plane waves across a single interface, are not suitable for thin-bed problems (Pan and Kristopher, 2013). Thin-bed seismic responses are composed of the superposition of all reflecting waveforms and multiples, including converted waves, which is different from a single-interface case (Chen and Liu, 2006).

Thin-bed reflections depend not only upon the elastic parameters of layered media, but also upon the thin-bed thickness and the frequency of incident waves. Brekhovskikh (1960) studies reflections and transmissions of plane waves propagating in layered media by elastic dynamic theory and derives accurate equations with displacement potential function. However, the complex propagator matrix limited their application in seismic inversion. Meissner and Meixner (1969) present the time delayed transmission/reflection method and deduce thin-bed reflection coefficients by multiplying the reflecting and transmitting coefficients of the top and bottom interfaces. Widess (1973) studies the normal pulse reflections from the top and bottom of a thin bed under the assumptions of equal amplitudes and opposite polarities and tries to predict thinbed thickness by amplitude information. Chung and Lawton (1995) extend Widess (1973) study into a thin bed, which has equal amplitudes and identical polarities in the bottom and top interfaces and analyze the influence of different wavelets on thin-bed reflections. Liu and Schmitt (2003) present an acoustic reflectance spectrum formula of a thin bed in the frequency domain and discuss the impact of thickness and Poisson's ratio on thin-bed seismic AVO

Manuscript received by the Editor 3 July 2015; revised manuscript received 22 March 2016; published online 27 July 2016.

${ }^{1}$ China University of Geosciences, School of Geophysics and Information Technology, Beijing, China. E-mail: yangchunanhui@163.com; yunwang@mail. iggcas.ac.cn.

${ }_{2}^{2}$ Imperial College London, Department of Earth Science and Engineering, Centre for Reservoir Geophysics, London, UK. E-mail: yanghua.wang@imperial. ac.uk

(C) 2016 Society of Exploration Geophysicists. All rights reserved. 
responses. Pan and Kristopher (2013) compare thin-bed elastic AVO responses with Liu and Schmitt's (2003) acoustic AVO responses, and they investigate the influence of quality factor $Q$ to examine attenuation effects.

The works above gave several description methods of thin-bed seismic responses. However, these reflection coefficient formulas are either too complex for application (Brekhovskikh, 1960; Meissner and Meixner, 1969) or only suitable to some extreme hypothetical conditions without considering multiples (Widess, 1973; Chung and Lawton, 1995; Liu and Schmitt, 2003; Pan and Kristopher 2013). In this paper, we initially give the propagator matrix equations for a thin-bed model with displacement functions, different from Brekhovskikh's derivation with displacement potential functions. Then, we simplify reflection and transmission coefficients of a thin bed in case of incident P-wave. We establish a quasi-Zoeppritz matrix approximation under the assumption that the middle layer is very thin by modeling a thin middle layer in homogeneous elastic three-layer media. Finally, we simulate different types of single thin-bed models and a well log containing thin layers, and discuss the precision of approximate equations for different thin-bed thicknesses and seismic responses of the well log.

\section{THIN-BED REFLECTION/TRANSMISSION COEFFICIENTS}

For an isotropic elastic model with two horizontal interfaces (Figure 1), according to boundary conditions, the thin-bed reflection and transmission coefficients defined by displacement with incident P-wave can be described as

$$
\mathbf{M}\left[\begin{array}{c}
R_{\mathrm{PP}} \\
R_{\mathrm{PS}} \\
T_{\mathrm{PP}} \\
T_{\mathrm{PS}}
\end{array}\right]=\mathbf{n},
$$

where $\mathbf{M}$ is a $4 \times 4$ matrix with four column vectors, $\mathbf{M} \equiv\left[\begin{array}{llll}\mathbf{m}_{1} & \mathbf{m}_{2} & \mathbf{m}_{3} & \mathbf{m}_{4}\end{array}\right]$, defined explicitly as

$$
\mathbf{m}_{1}=-\mathbf{A}\left[\begin{array}{c}
\sin \alpha_{1} \\
-\cos \alpha_{1} \\
-j \rho_{1} \omega V_{\mathrm{P} 1} \cos 2 \beta_{1} \\
j \rho_{1} \omega \frac{V_{\mathrm{S} 1}^{2}}{V_{\mathrm{P} 1}} \sin 2 \alpha_{1}
\end{array}\right]
$$

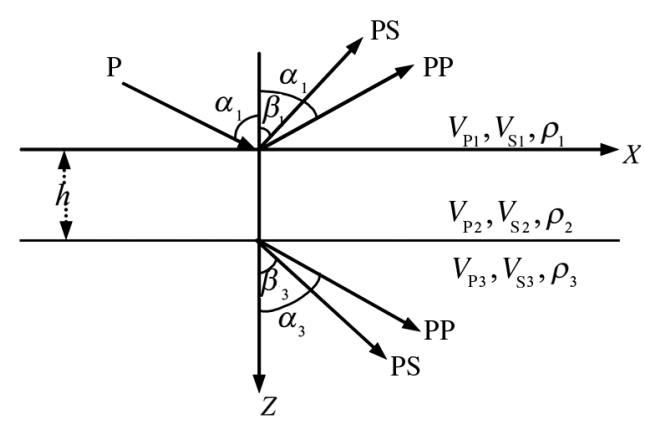

Figure 1. A thin-bed model with two horizontal interfaces.

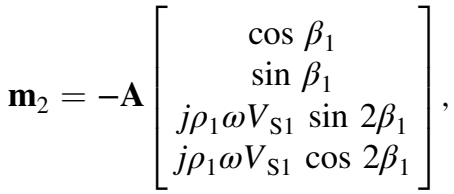

$$
\begin{aligned}
& \mathbf{m}_{3}=\left[\begin{array}{c}
\sin \alpha_{3} \\
\cos \alpha_{3} \\
-j \rho_{3} \omega V_{\mathrm{P} 3} \cos 2 \beta_{3} \\
-j \rho_{3} \omega \frac{V_{\mathrm{S} 3}^{2}}{V_{\mathrm{P} 3}} \sin 2 \alpha_{3}
\end{array}\right], \\
& \mathbf{m}_{4}=\left[\begin{array}{c}
\cos \beta_{3} \\
-\sin \beta_{3} \\
j \rho_{3} \omega V_{\mathrm{S} 3} \sin 2 \beta_{3} \\
-j \rho_{3} \omega V_{\mathrm{S} 3} \cos 2 \beta_{3}
\end{array}\right], \\
& \mathbf{n}=-\mathbf{A}\left[\begin{array}{c}
-\sin \alpha_{1} \\
-\cos \alpha_{1} \\
j \rho_{1} \omega V_{\mathrm{P} 1} \cos 2 \beta_{1} \\
j \rho_{1} \omega \frac{V_{\mathrm{S} 1}^{2}}{V_{\mathrm{P} 1}} \sin 2 \alpha_{1}
\end{array}\right],
\end{aligned}
$$

$\mathbf{n}$ is a $4 \times 1$ vector, $\mathbf{A}$ is $4 \times 4$ matrix with elements $a_{i j}$ defined as

$$
\begin{aligned}
& a_{11}=a_{44}=2 \sin ^{2} \beta_{2} \cos P+\cos 2 \beta_{2} \cos Q, \\
& a_{12}=a_{34}=-j\left(\tan \alpha_{2} \cos 2 \beta_{2} \sin P-\sin 2 \beta_{2} \sin Q\right) \text {, } \\
& a_{13}=a_{24}=\frac{j \sin \alpha_{2}}{\rho_{2} \omega V_{\mathrm{P} 2}}(\cos P-\cos Q) \text {, } \\
& a_{14}=\frac{1}{\rho_{2} \omega V_{\mathrm{S} 2}}\left(\tan \alpha_{2} \sin \beta_{2} \sin P+\cos \beta_{2} \sin Q\right) \text {, } \\
& a_{21}=a_{43}=-j\left(\frac{V_{\mathrm{S} 2} \cos \alpha_{2}}{V_{\mathrm{P} 2} \cos \beta_{2}} \sin 2 \beta_{2} \sin P\right. \\
& \left.-\tan \beta_{2} \cos 2 \beta_{2} \sin Q\right) \text {, } \\
& a_{22}=a_{33}=\cos 2 \beta_{2} \cos P+2 \sin ^{2} \beta_{2} \cos Q \text {, } \\
& a_{23}=\frac{1}{\rho_{2} \omega V_{\mathrm{P} 2}}\left(\cos \alpha_{2} \sin P+\tan \beta_{2} \sin \alpha_{2} \sin Q\right) \text {, } \\
& a_{31}=a_{42}=2 j \rho_{2} \omega V_{\mathrm{S} 2} \sin \beta_{2} \cos 2 \beta_{2}(\cos Q-\cos P) \text {, } \\
& a_{32}=-\rho_{2} \omega\left(\frac{V_{\mathrm{P} 2} \cos ^{2} 2 \beta_{2}}{\cos \alpha_{2}} \sin P\right. \\
& \left.+4 V_{\mathrm{S} 2} \cos \beta_{2} \sin ^{2} \beta_{2} \sin Q\right) \text {, } \\
& a_{41}=-\rho_{2} \omega V_{\mathrm{S} 2}\left(\frac{4 V_{\mathrm{S} 2} \sin ^{2} \beta_{2} \cos \alpha_{2}}{V_{\mathrm{P} 2}} \sin P\right. \\
& \left.+\frac{\cos ^{2} 2 \beta_{2}}{\cos \beta_{2}} \sin Q\right) \text {, }
\end{aligned}
$$


and

$$
P=\frac{\omega h}{V_{\mathrm{P} 2}} \cos \alpha_{2}, \quad Q=\frac{\omega h}{V_{\mathrm{S} 2}} \cos \beta_{2} .
$$

In equation $2, V_{\mathrm{P}}$ and $V_{\mathrm{S}}$ are the $\mathrm{P}$ - and $\mathrm{S}$-wave velocities, respectively, $\rho$ is the density, $\alpha$ is the $\mathrm{P}$-wave incident, transmitted, or reflected angle, $\beta$ is the $S$-wave transmitted or reflected angle, and their subscripts $i=1,2,3$ refer to three layers, $h$ is the thickness of the middle layer $(i=2), \omega$ is the angular frequency, and $j=\sqrt{-1}$ is the imaginary symbol.

It can be proved that matrix $\mathbf{M}$ is in full rank. Thus, according to Cramer's rule, the reflection and transmission coefficients $R_{\mathrm{PP}}, R_{\mathrm{PS}}$, $T_{\mathrm{PP}}$, and $T_{\mathrm{PS}}$ can be obtained.

Note that the reflection and transmission coefficients $R_{\mathrm{PP}}, R_{\mathrm{PS}}$, $T_{\mathrm{PP}}$, and $T_{\mathrm{PS}}$ in equation 1 are defined in terms of displacements. They are different from reflection and transmission coefficients described with displacement potential functions (Brekhovskikh, 1960). Meanwhile, when the thickness of the middle layer is equal to zero, equation 1 can be simplified into the Zoeppritz equations.

\section{QUASI-ZOEPPRITZ MATRIX FORM}

When the middle layer's thickness is thin, $h \ll V_{\mathrm{P} 2} / \omega$ and $h \ll V_{\mathrm{S} 2} / \omega$, then we have $P \ll 1$ and $Q \ll 1$ in equation 2 . Meanwhile, we can make additional approximations using the following first-order Taylor expansions:

$\sin P \approx P, \quad \sin Q \approx Q, \quad \cos P \approx 1, \quad \cos Q \approx 1$,

which simplify the elements $a_{i j}$ in matrix $\mathbf{A}$ as

$$
\begin{aligned}
& a_{11}=a_{44}=a_{22}=a_{33}=1, \\
& a_{12}=a_{34}=j \omega h \frac{\sin \alpha_{1}}{V_{\mathrm{P} 1}}, \\
& a_{13}=a_{31}=a_{24}=a_{42}=0, \\
& a_{14}=\frac{h}{\rho_{2} V_{\mathrm{S} 2}^{2}}, \\
& a_{21}=a_{43}=-j \omega h\left(2 \frac{V_{\mathrm{S} 2}^{2}}{V_{\mathrm{P} 2}^{2}}-1\right) \frac{\sin \alpha_{1}}{V_{\mathrm{P} 1}}, \\
& a_{23}=\frac{h}{\rho_{2} V_{\mathrm{P} 2}^{2}}, \\
& a_{32}=-\rho_{2} \omega^{2} h, \\
& a_{41}=-\rho_{2} \omega^{2} h\left[1-4 V_{\mathrm{S} 2}^{2}\left(1-\frac{V_{\mathrm{S} 2}^{2}}{V_{\mathrm{P} 2}^{2}}\right) \frac{\sin ^{2} \alpha_{1}}{V_{\mathrm{P} 1}^{2}}\right],
\end{aligned}
$$

according to Snell's law. Obviously, all coefficients in equation 4 depend on only the P-wave incident angle $\alpha_{1}$.

Using these simplified $a_{i j}$ coefficients, all of the column vectors from equations $2 \mathrm{a}$ to $2 \mathrm{e}$ can also be represented in terms of the $\mathrm{P}$-wave incident angle. This allows equation 1 to be modified to represent variation with respect to the incident angle, making it suitable for direct AVO analysis.
For the first-column vector $\mathbf{m}_{1} \equiv\left[m_{i 1}\right], i=1, \ldots, 4$, we have

$$
\begin{aligned}
m_{11}= & -\sin \alpha_{1}+j \frac{\omega h}{V_{\mathrm{P} 1}}\left(\frac{1}{2}-\frac{\rho_{1}}{\rho_{2}} \frac{V_{\mathrm{S} 1}^{2}}{V_{\mathrm{S} 2}^{2}}\right) \sin 2 \alpha_{1}, \\
m_{21}= & \cos \alpha_{1}+j \frac{\omega h}{V_{\mathrm{P} 1}}\left[\frac{\rho_{1}}{\rho_{2}} \frac{V_{\mathrm{P} 1}^{2}}{V_{\mathrm{P} 2}^{2}}\right. \\
& \left.-\left(1-2 \frac{V_{\mathrm{S} 2}^{2}}{V_{\mathrm{P} 2}^{2}}+2 \frac{\rho_{1}}{\rho_{2}} \frac{V_{\mathrm{S} 1}^{2}}{V_{\mathrm{P} 2}^{2}}\right) \sin ^{2} \alpha_{1}\right], \\
m_{31}= & \left(1-2 \frac{V_{\mathrm{S} 1}^{2}}{V_{\mathrm{P} 1}^{2}} \sin ^{2} \alpha_{1}\right) \\
& +j \frac{\omega h}{V_{\mathrm{P} 1}}\left(\frac{\rho_{2}}{\rho_{1}} \cos \alpha_{1}-\frac{V_{\mathrm{S} 1}^{2}}{V_{\mathrm{P} 1}^{2}} \sin 2 \alpha_{1} \sin \alpha_{1}\right), \\
m_{41}= & \sin 2 \alpha_{1} \\
& +j \frac{\omega h}{V_{\mathrm{P} 1}}\left[\frac{\rho_{2}}{\rho_{1}} \frac{V_{\mathrm{P} 1}^{2}}{V_{\mathrm{S} 1}^{2}}+4 \frac{\rho_{2}}{\rho_{1}} \frac{V_{\mathrm{S} 2}^{2}}{V_{\mathrm{S} 1}^{2}}\left(\frac{V_{\mathrm{S} 2}^{2}}{V_{\mathrm{P} 2}^{2}}-1\right) \sin ^{2} \alpha_{1}\right. \\
& \left.+\left(2 \frac{V_{\mathrm{S} 2}^{2}}{V_{\mathrm{P} 2}^{2}}-1\right)\left(\frac{V_{\mathrm{P} 1}^{2}}{V_{\mathrm{S} 1}^{2}}-2 \sin ^{2} \alpha_{1}\right)\right] \sin ^{2} .
\end{aligned}
$$

For the second-column vector $\mathbf{m}_{2} \equiv\left[m_{i 2}\right]$, we have

$$
\begin{aligned}
m_{12}= & -\left(1-\frac{V_{\mathrm{S} 1}^{2}}{V_{\mathrm{P} 1}^{2}} \sin ^{2} \alpha_{1}\right)^{1 / 2} \\
& -j \frac{\omega h}{V_{\mathrm{P} 1}} \frac{V_{\mathrm{S} 1}}{V_{\mathrm{P} 1}}\left[\frac{\rho_{1}}{\rho_{2}} \frac{V_{\mathrm{P} 1}^{2}}{V_{\mathrm{S} 2}^{2}}+\left(1-2 \frac{\rho_{1}}{\rho_{2}} \frac{V_{\mathrm{S} 1}^{2}}{V_{\mathrm{S} 2}^{2}}\right) \sin ^{2} \alpha_{1}\right], \\
m_{22}= & -\frac{V_{\mathrm{S} 1}}{V_{\mathrm{P} 1}} \sin \alpha_{1}-j \frac{\omega h}{V_{\mathrm{P} 1}}\left(1-2 \frac{V_{\mathrm{S} 2}^{2}}{V_{\mathrm{P} 2}^{2}}+2 \frac{\rho_{1}}{\rho_{2}} \frac{V_{\mathrm{S} 1}^{2}}{V_{\mathrm{P} 2}^{2}}\right) \\
& \times\left(1-\frac{V_{\mathrm{S} 1}^{2}}{V_{\mathrm{P} 1}^{2}} \sin ^{2} \alpha_{1}\right)^{1 / 2} \sin \alpha_{1}, \\
m_{32}= & -2 \frac{V_{\mathrm{S} 1}^{2}}{V_{\mathrm{P} 1}^{2}}\left(1-\frac{V_{\mathrm{S} 1}^{2}}{V_{\mathrm{P} 1}^{2}} \sin ^{2} \alpha_{1}\right)^{1 / 2} \sin ^{\alpha_{1}} \\
& -j \frac{\omega h}{V_{\mathrm{P} 1}} \frac{V_{\mathrm{S} 1}}{V_{\mathrm{P} 1}}\left(1+\frac{\rho_{2}}{\rho_{1}}-2 \frac{V_{\mathrm{S} 1}^{2}}{V_{\mathrm{P} 1}^{2}} \sin ^{2} \alpha_{1}\right) \sin ^{\alpha_{1},} \\
m_{42}= & V_{\mathrm{P} 1}\left(1-2 \frac{V_{\mathrm{S} 1}^{2}}{V_{\mathrm{S} 1}^{2}} \sin ^{2} \alpha_{1}\right) \\
& +j \frac{\omega h}{V_{\mathrm{P} 1}}\left[\frac{\rho_{2}}{\rho_{1}} \frac{V_{\mathrm{P} 1}^{2}}{V_{\mathrm{S} 1}^{2}}+4 \frac{\rho_{2}}{\rho_{1}} \frac{V_{\mathrm{S} 2}^{2}}{V_{\mathrm{S} 1}^{2}}\left(\frac{V_{\mathrm{S} 2}^{2}}{V_{\mathrm{P} 2}^{2}}-1\right) \sin ^{2} \alpha_{1}\right. \\
& \left.-2\left(\frac{2 V_{\mathrm{S} 2}^{2}}{V_{\mathrm{P} 2}^{2}}-1\right)^{2} \sin ^{2} \alpha_{1}\right]\left(1-\frac{V_{\mathrm{S} 1}^{2}}{V_{\mathrm{P} 1}^{2}} \sin ^{2} \alpha_{1}\right)^{1 / 2} .
\end{aligned}
$$

For the third-column vector $\mathbf{m}_{3}$ and the fourth-column vector $\mathbf{m}_{4}$, we have

$$
\mathbf{m}_{3}=\left[\begin{array}{c}
\frac{V_{\mathrm{P} 3}}{V_{\mathrm{P}}} \sin \alpha_{1} \\
\left(1-\frac{V_{\mathrm{P} 3}^{2}}{V_{\mathrm{P} 1}^{2}} \sin ^{2} \alpha_{1}\right)^{1 / 2} \\
-\frac{\rho_{3}}{\rho_{1}} \frac{V_{\mathrm{P} 3}}{V_{\mathrm{P} 1}}\left(1-2 \frac{V_{\mathrm{S} 3}^{2}}{V_{\mathrm{P} 1}^{2}} \sin ^{2} \alpha_{1}\right) \\
2 \frac{\rho_{3}}{\rho_{1}} \frac{V_{\mathrm{S} 3}^{2}}{V_{\mathrm{S} 1}^{2}}\left(1-\frac{V_{\mathrm{P} 3}^{2}}{V_{\mathrm{P} 1}^{2}} \sin ^{2} \alpha_{1}\right)^{1 / 2} \sin \alpha_{1}
\end{array}\right],
$$




$$
\mathbf{m}_{4}=\left[\begin{array}{c}
\left(1-\frac{V_{\mathrm{S} 3}^{2}}{V_{\mathrm{P} 1}^{2}} \sin ^{2} \alpha_{1}\right)^{1 / 2} \\
-\frac{V_{\mathrm{S} 3}}{V_{\mathrm{P} 1}} \sin \alpha_{1} \\
2 \frac{\rho_{3}}{\rho_{1}} \frac{V_{\mathrm{S} 3}^{2}}{V_{\mathrm{P} 1}^{2}}\left(1-\frac{V_{\mathrm{S} 3}^{2}}{V_{\mathrm{P} 1}^{2}} \sin ^{2} \alpha_{1}\right)^{1 / 2} \sin \alpha_{1} \\
\frac{\rho_{3}}{\rho_{1}} \frac{V_{\mathrm{S} 3} V_{\mathrm{P} 1}}{V_{\mathrm{S} 1}^{2}}\left(1-2 \frac{V_{\mathrm{S} 3}^{2}}{V_{\mathrm{P} 1}^{2}} \sin ^{2} \alpha_{1}\right)
\end{array}\right] .
$$

For the right column vector $\mathbf{n} \equiv\left[n_{i}\right]$, we have

$$
\begin{aligned}
n_{1}= & \sin \alpha_{1}+j \frac{\omega h}{V_{\mathrm{P} 1}}\left(\frac{1}{2}-\frac{\rho_{1}}{\rho_{2}} \frac{V_{\mathrm{S} 1}^{2}}{V_{\mathrm{S} 2}^{2}}\right) \sin 2 \alpha_{1}, \\
n_{2}= & \cos \alpha_{1}+j \frac{\omega h}{V_{\mathrm{P} 1}}\left[\left(1-2 \frac{V_{\mathrm{S} 2}^{2}}{V_{\mathrm{P} 2}^{2}}+2 \frac{\rho_{1}}{\rho_{2}} \frac{V_{\mathrm{S} 1}^{2}}{V_{\mathrm{P} 2}^{2}}\right) \sin ^{2} \alpha_{1}-\frac{\rho_{1}}{\rho_{2}} \frac{V_{\mathrm{P} 1}^{2}}{V_{\mathrm{P} 2}^{2}}\right], \\
n_{3}= & -\left(1-2 \frac{V_{\mathrm{S} 1}^{2}}{V_{\mathrm{P} 1}^{2}} \sin ^{2} \alpha_{1}\right) \\
& +j \frac{\omega h}{V_{\mathrm{P} 1}}\left(\frac{\rho_{2}}{\rho_{1}} \cos \alpha_{1}+\frac{V_{\mathrm{S} 1}^{2}}{V_{\mathrm{P} 1}^{2}} \sin 2 \alpha_{1} \sin \alpha_{1}\right), \\
n_{4}= & \sin 2 \alpha_{1}-j \frac{\omega h}{V_{\mathrm{P} 1}}\left[4 \frac{\rho_{2}}{\rho_{1}} \frac{V_{\mathrm{S} 2}^{2}}{V_{\mathrm{S} 1}^{2}}\left(\frac{V_{\mathrm{S} 2}^{2}}{V_{\mathrm{P} 2}^{2}}-1\right) \sin ^{2} \alpha_{1}\right. \\
& \left.+\frac{\rho_{2}}{\rho_{1}} \frac{V_{\mathrm{P} 1}^{2}}{V_{\mathrm{S} 1}^{2}}+\left(2 \frac{V_{\mathrm{S} 2}^{2}}{V_{\mathrm{P} 2}^{2}}-1\right)\left(\frac{V_{\mathrm{P} 1}^{2}}{V_{\mathrm{S} 1}^{2}}-2 \sin ^{2} \alpha_{1}\right)\right] \sin \alpha_{1} .
\end{aligned}
$$

Note that the elements in vectors $\mathbf{m}_{1}, \mathbf{m}_{2}$, and $\mathbf{n}$ are complex valued, and the elements in vectors $\mathbf{m}_{3}$ and $\mathbf{m}_{4}$, which are the weights of transmission coefficients, are real valued. Comparison of the vectors above shows that the factor thickness causes thin beds to have complex reflection coefficients and complex characteristic parameters, such as peak instantaneous frequency or the amplitude spectrum. These parameters can be used in estimation of thin-bed thicknesses (Liu and Marfurt, 2006; Puryear and Castagna, 2008).

Under the assumption that the middle layer is thin, equation 1 for thin-bed reflection-transmission coefficients is simplified into a relatively simple form, which is similar to the Zoeppritz equations. Equation 5 contains the plane-wave reflection and transmission coefficients, which are frequency dependent. They also depend on the thickness and elastic parameters of the thin bed.

In addition, it is worthwhile to note that the reflection and transmission coefficient equations that Schoenberg and Protazio (1992) give for anisotropic layered media may also be simplified into equation 5 in the case of homogeneous elastic media under the same thin-thickness assumption.

\section{APPROXIMATION ACCURACY OF SINGLE THIN-BED MODELS}

To verify the accuracy and applicability of quasi-Zoeppritz matrix approximation, we test first on single thin-bed models and analyze errors in amplitude and phase for reflection coefficients' approximation.

There are four different types of single thin-bed models: (1) highimpedance thin bed $\left(\rho_{1} V_{1}<\rho_{2} V_{2}>\rho_{3} V_{3}\right)$, (2) low-to-high impedance transition layers $\left(\rho_{1} V_{1}<\rho_{2} V_{2}<\rho_{3} V_{3}\right)$, (3) low-impedance thin bed $\left(\rho_{1} V_{1}>\rho_{2} V_{2}<\rho_{3} V_{3}\right)$, and (4) high-to-low impedance transition layers $\left(\rho_{1} V_{1}>\rho_{2} V_{2}>\rho_{3} V_{3}\right)$. Table 1 lists the elastic parameters associated with these four models (Liu and Schmitt, 2003; Chen and Liu, 2006). It can be calculated that models 1 and 3 would produce opposite-polarity reflections, whereas models 2 and 4 would produce identical-polarity reflections (Kallweit and Wood, 1982).

When the normalized impedance difference between two sides of an interface is calculated by

$$
\Delta I=\frac{\rho_{i} V_{i}-\rho_{i-1} V_{i-1}}{\rho_{i} V_{i}+\rho_{i-1} V_{i-1}},
$$

where $i=2,3$, representing the normal incident reflectivity, the Pwave normalized impedance differences of the four models' top and bottom interfaces are also listed in Table 1. All of these models have strong impedance differences, for which linear approximations (Aki and Richards, 1980; Shuey, 1985) may lead to significant errors; however, quadratic or quartic approximations, proposed by Wang (1999), could have a high accuracy.

According to Widess (1973), a thin bed is one whose thickness is less than approximately $\lambda / 8$, where $\lambda$ is the wavelength corresponding to the dominant frequency in the thin bed. In our numerical calculation, the thin-bed thicknesses are set as variable from $\lambda / 8$ to $\lambda / 60$. Note that PS-waves have lower frequency than PP-waves in field seismic data (Wang et al., 2009), and the former have lower velocities than the latter for the same layer. We test PP- and PS-reflection coefficients with the same ratios of the thin-bed thicknesses to their respective wavelengths. In seismic exploration of coal and gas-oil reservoirs, the reflections are mostly considered within incident angles smaller than the first critical angles, so we set the critical angles as the maximum incident angles, which are $30^{\circ}, 30^{\circ}$, and $46.16^{\circ}$ for models $1-3$, respectively. Although model 4 does not have the critical angle, we take $89^{\circ}$ as its maximum incident angle. Figures 2, 3, 4, and 5 plot the relative errors for each model, calculated as approximation/true -1 , in percentage, and Table 2 lists the maximum absolute values of these relation errors.

For model 1, errors in amplitude for the $R_{\mathrm{PP}}$ approximation are less than or equal to $-9.31 \%$, with the maximum error appeared in the case of $\lambda / 8$ thickness and vertical incidence (Figure $2 \mathrm{a}$ and

Table 1. Parameters of thin-bed models. Units of velocities and densities are $\mathrm{m} / \mathrm{s}$ and $\mathrm{g} / \mathrm{cm}^{3}$, respectively.

\begin{tabular}{lcccccccccc}
\hline & $V_{\mathrm{P} 1}$ & $V_{\mathrm{S} 1}$ & $\rho_{1}$ & $V_{\mathrm{P} 2}$ & $V_{\mathrm{S} 2}$ & $\rho_{2}$ & $V_{\mathrm{P} 3}$ & $V_{\mathrm{S} 3}$ & $\rho_{3}$ & $\left(\Delta I_{\text {top }}, \Delta I_{\text {bottom }}\right)$ \\
\hline Model 1 & 3050 & 1525 & 2.7 & 6100 & 3050 & 2.7 & 2500 & 1525 & 2.7 & $(0.3333,-0.4186)$ \\
Model 2 & 3050 & 1600 & 2.7 & 4200 & 2500 & 2.7 & 6100 & 3100 & 2.7 & $(0.1586,0.1845)$ \\
Model 3 & 2200 & 1200 & 2.3 & 1500 & 800 & 2.2 & 3050 & 1400 & 2.35 & $(-0.2105,0.3695)$ \\
Model 4 & 6100 & 3100 & 2.7 & 4200 & 2500 & 2.7 & 3050 & 1600 & 2.7 & $(-0.1845,-0.1836)$ \\
\hline
\end{tabular}


Table 2). As thin-bed thickness decreases and incident angle increases, approximation errors decline quickly to less than $5 \%$, and trend toward to zero. For the $R_{\mathrm{PS}}$ approximation, errors in amplitude are less than or equal to $-5.91 \%$ with thickness less than $\lambda / 10$. Similarly, the errors decline with decreasing thickness and increasing angles. The PS-wave approximation shows higher errors with the same ratios of the thin-bed thicknesses to their respective wavelengths and quicker reduction with increasing incident angles. Errors in phase for the $R_{\mathrm{PP}}$ approximation are larger than $10 \%$ for the $\lambda / 8$ and $\lambda / 10$ cases and not acceptable, and smaller than $5 \%$ for all other (thinner) thicknesses (Figure 2b). For the $R_{\mathrm{PS}}$ approximation, errors in phase decline from more than $9.04 \%(\lambda / 20)$ to approximately $3.49 \%(\lambda / 60)$. The $R_{\mathrm{PS}}$ approximation is inapplicable for the $\lambda / 8$ and $\lambda / 10$ cases because of large errors $(>10 \%)$.
For model 2, errors in amplitude for the $R_{\mathrm{PP}}$ approximation are less than $5 \%$ except in the case of $\lambda / 8$ thickness (Figure $3 a$ ). When the thickness is $\lambda / 8$, errors are slightly more than $10 \%$ for normal incidence, but decrease very quickly with increasing incident angles. For the $R_{\mathrm{PS}}$ approximation, errors in amplitude are less than $2.42 \%$ with the incident angles less than $27^{\circ}$. The approximation accuracy of $R_{\mathrm{PP}}$ is higher than that of $R_{\mathrm{PS}}$ for the same ratios of layer thickness to their wavelengths except the case of $\lambda / 8$ (Table 2). When the incident angle is near the critical angle, PS-wave errors increase quickly, though are still smaller than $8.24 \%$. Errors in phase for the $R_{\mathrm{PP}}$ and $R_{\mathrm{PS}}$ approximation are smaller than $5 \%$ except the cases of $\lambda / 8$ and $\lambda / 10$ for PP-wave. In amplitude and phase, errors for the $R_{\mathrm{PS}}$ approximation change quickly near the critical angle.

Table 2. The maximum absolute values of the relative errors $(\%)$ of thin-bed models with the incident angles smaller than the first critical angles (when they exist). "Amp" stands for amplitude.

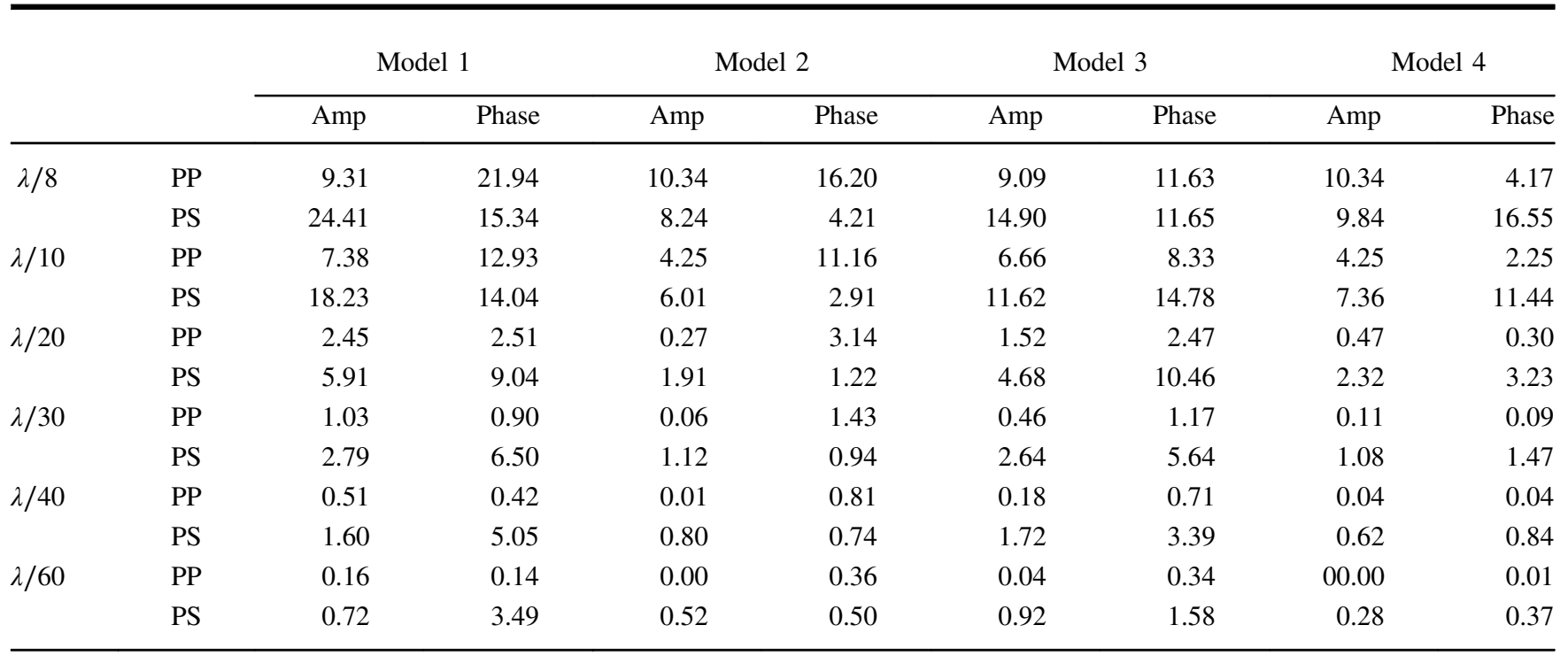
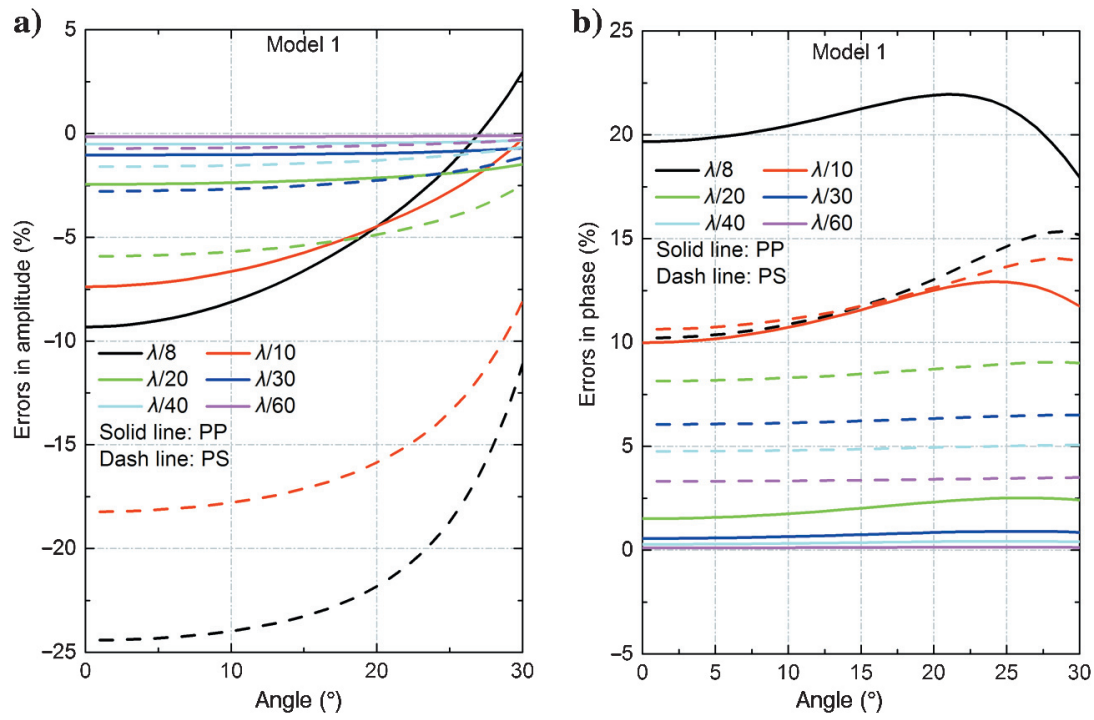

Figure 2. Errors for reflection coefficients' approximation of model 1. (a) Errors in amplitude and (b) errors in phase. 
Figure 3. Errors for reflection coefficients' approximation of model 2. (a) Errors in amplitude and (b) errors in phase.

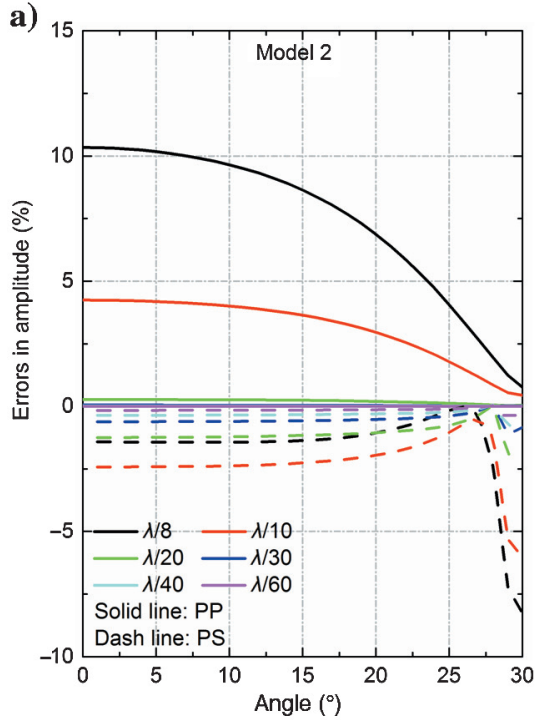
mation of model 3. (a) Errors in amplitude and
Figure 4. Errors for reflection coefficients' approxi(b) errors in phase.
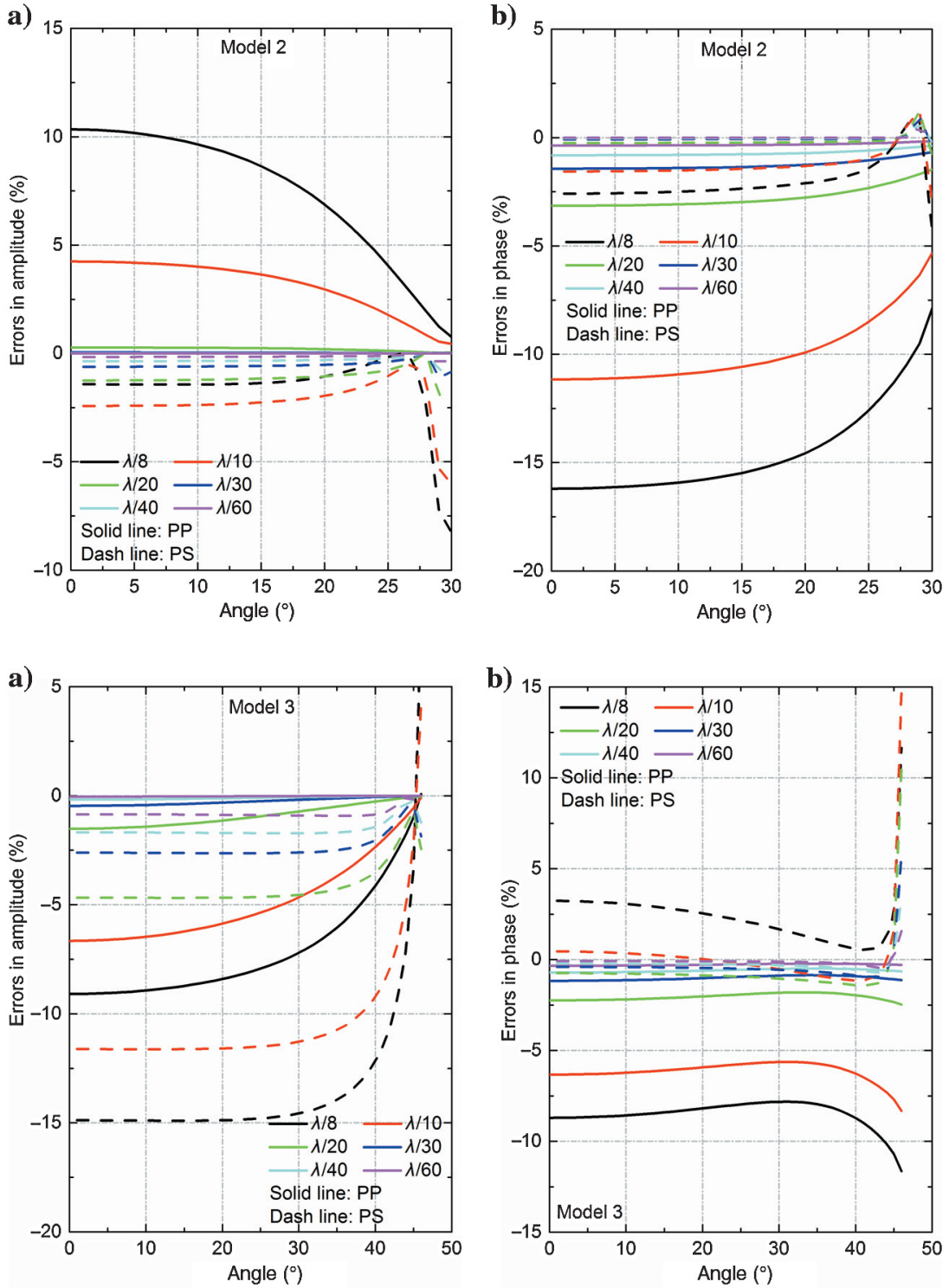

Figure 5. Errors for reflection coefficients' approximation of model 4. (a) Errors in amplitude and (b) errors in phase. a)

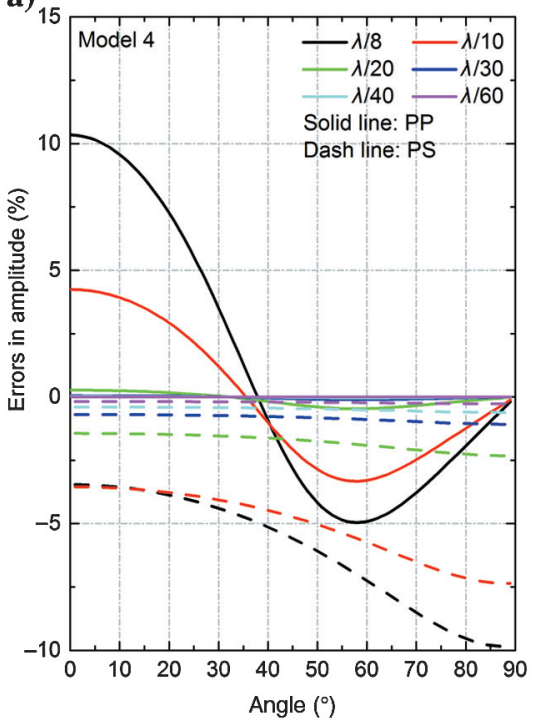

b)

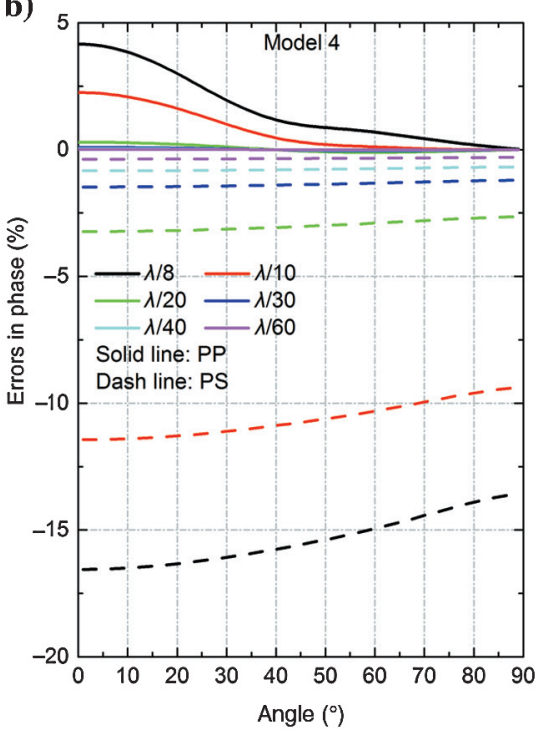


For model 3, errors in amplitude for the $R_{\mathrm{PP}}$ approximation are less than or equal to $-9.09 \%$, and decrease in magnitude with decreasing thickness and increasing incident angle. For the thicknesses less than or equal to $\lambda / 20$, approximation errors are less than $-1.52 \%$ (Figure $4 \mathrm{a}$ ). For the $R_{\mathrm{PS}}$ approximation, errors in amplitude are not sensitive to the incident angles except around the critical angle and follow the same trend - errors decrease as thickness decreases. Except for the thicknesses of $\lambda / 8$ and $\lambda / 10$, where errors remain approximately $-14.90 \%$ and $-11.62 \%$, respectively, errors of the other thicknesses are smaller than $5 \%$. Errors in phase for the $R_{\mathrm{PP}}$ approximation are less than $10 \%$ except around the critical angle for the $\lambda / 8$ and $\lambda / 10$ cases, and smaller than $2.47 \%$ for the other thicknesses. For the $R_{\mathrm{PS}}$ approximation, errors in phase are less than $3.23 \%$ except around the critical angle (Figure $4 \mathrm{~b}$ ). When the incident angles are near the critical angle, the PS-wave approximation shows a sharp increase in error.

For model 4, errors in amplitude for the $R_{\mathrm{PP}}$ approximation are less than $4.25 \%$ except the case of $\lambda / 8$ (Figure $5 \mathrm{a}$ ). When the thickness is $\lambda / 8$, errors are slightly more than $10 \%$ for normal incidence, but decline very quickly to less than $5 \%$. Errors in amplitude for the $R_{\mathrm{PS}}$ approximation are less than $5 \%$ when the thicknesses are less than or equal to $\lambda / 20$. Errors in phase for the $R_{\mathrm{PP}}$ approximation are smaller than $4.17 \%$ (Figure $5 \mathrm{~b}$ ). For PS-wave, errors in phase are less than $3.23 \%$ for cases of thicknesses less than or equal to $\lambda / 20$. Except amplitude approximation near normal incidence, the $R_{\mathrm{PS}}$ approximation fails for the $\lambda / 8$ and $\lambda / 10$ cases because of large errors $(>10 \%)$.

To analyze the influence of impedance differences on approximation accuracy, we present another series of thin-bed models with opposite polarity but gradually decreased impedance differences. Model parameters and P-wave normalized impedance differences of thin-bed top and bottom interfaces are listed in Table 3.

For this group of models, errors in amplitude for the $R_{\mathrm{PP}}$ and $R_{\mathrm{PS}}$ approximation decrease with decreasing impendence difference, except for incident angles near the critical angle. The approximation accuracy of $R_{\mathrm{PP}}$ is higher than that of $R_{\mathrm{PS}}$ for the same ratios of thickness to their wavelengths. Taking thickness equal to $1 / 20$ wavelengths, for example (as seen in Figure 6), the $R_{\mathrm{PP}}$ and $R_{\mathrm{PS}}$ approximation share a similar trend of reduced errors in amplitude with decreasing impedance difference, except for near critical angles.

In summary, the numerical tests reveal that

1) Approximation accuracy of the quasi-Zoeppritz matrix is high enough to be applied in seismic inversion of a single thin bed, and will be improved when the thickness of the middle layer and/or the impendence difference decrease. Errors for the $R_{\mathrm{PP}}$ approximation are generally less than $10 \%$ when the thin-bed thicknesses are smaller than one-eighth of PP-wavelengths, whereas errors for the $R_{\mathrm{PS}}$ approximation are relatively worse.

2) Comparing PP- and PS-waves with the same ratios of thin-bed thickness to their respective wavelengths, errors in amplitude for the $R_{\mathrm{PP}}$ approximation are smaller than those for the $R_{\mathrm{PS}}$ approximation except the case of $\lambda / 8$ for the thin-bed models with identical polarities.

3) Errors for the $R_{\mathrm{PS}}$ approximation increase quickly near the critical angles (when they exist) except in the case of a high-impedance difference (such as model 1), and the quasi-Zoeppritz matrix approximation is inapplicable.

4) Errors in phase for the $R_{\mathrm{PP}}$ and $R_{\mathrm{PS}}$ approximation are less than $10 \%$ for the cases of thicknesses less than $\lambda / 10$.

\section{APPROXIMATION ACCURACY OF SYNTHETIC SEISMOGRAMS}

To verify the acceptability of quasi-Zoeppritz matrix approximation on the actual seismic data, we test further on a well log con-

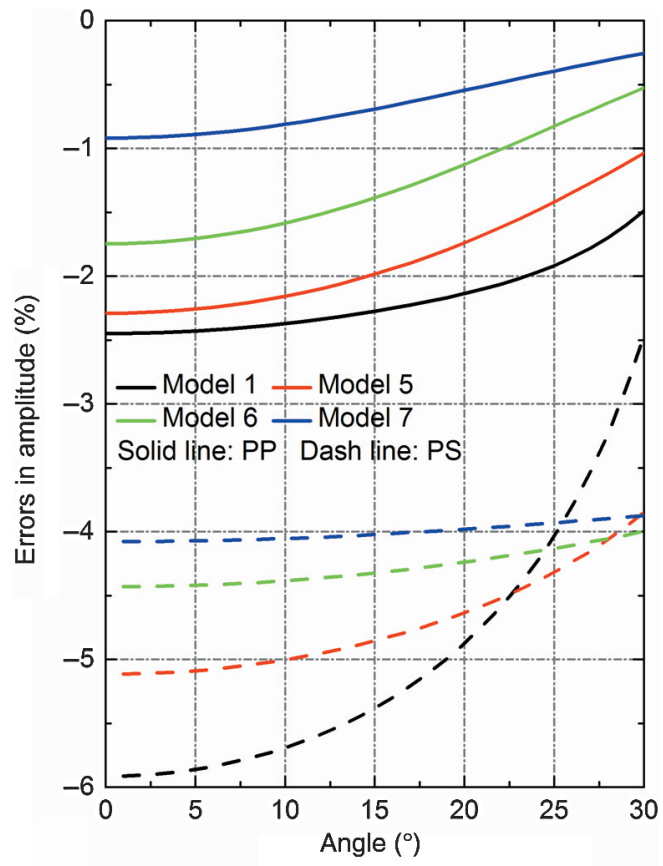

Figure 6. Errors in amplitude for reflection coefficients' approximation of thin-bed models with thickness equal to $\lambda / 20$, as impedance difference decreasing.

Table 3. Parameters of thin-bed models with opposite polarities and decreasing impedance differences. Units of velocities and densities are $\mathrm{m} / \mathrm{s}$ and $\mathrm{g} / \mathrm{cm}^{3}$, respectively.

\begin{tabular}{lcccccccccc} 
& $V_{\mathrm{P} 1}$ & $V_{\mathrm{S} 1}$ & $\rho_{1}$ & $V_{\mathrm{P} 2}$ & $V_{\mathrm{S} 2}$ & $\rho_{2}$ & $V_{\mathrm{P} 3}$ & $V_{\mathrm{S} 3}$ & $\rho_{3}$ & $\left(\Delta I_{\text {top }}, \Delta I_{\text {bottom }}\right)$ \\
\hline Model 1 & 3050 & 1525 & 2.7 & 6100 & 3050 & 2.7 & 2500 & 1525 & 2.7 & $(0.3333,-0.4186)$ \\
Model 5 & 3050 & 1525 & 2.7 & 5100 & 2550 & 2.7 & 2500 & 1525 & 2.7 & $(0.2515,-0.3421)$ \\
Model 6 & 3050 & 1525 & 2.7 & 4100 & 2050 & 2.7 & 2500 & 1525 & 2.7 & $(0.1468,-0.2424)$ \\
Model 7 & 3050 & 1525 & 2.7 & 3500 & 1750 & 2.7 & 2500 & 1525 & 2.7 & $(0.0687,-0.1666)$ \\
\hline
\end{tabular}


Figure 7. A well log containing thin layers from a coal mine in China. (a) Before well-logging curves stratification and (b) after well-logging curves stratification. Dashed green frame indicates the position of a thin coal seam.

Figure 8. PP-wave synthetic seismograms of the well log. (a) The PP-wave seismic response simulated by the exact formula and (b) PP-wave seismic response simulated by the approximation formula. The red arrows indicate PP-wave seismic responses of the thin coal seam.

Figure 9. PS-wave synthetic seismograms of the well log. (a) The PS-wave seismic response simu-

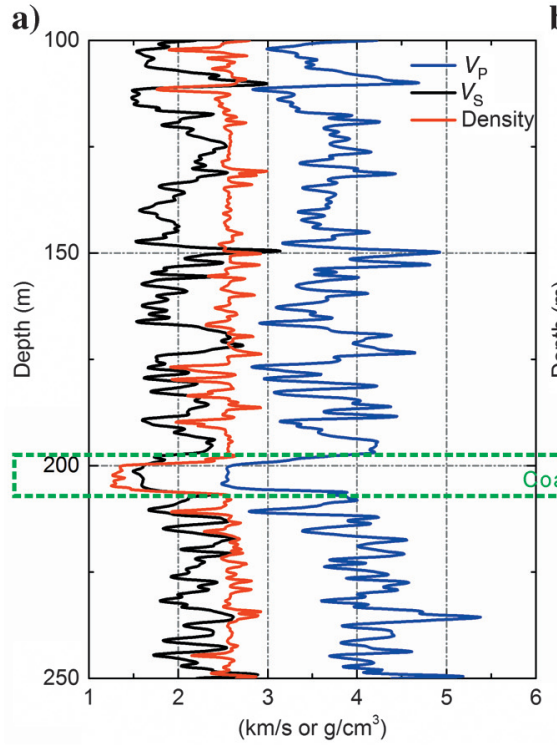

b)

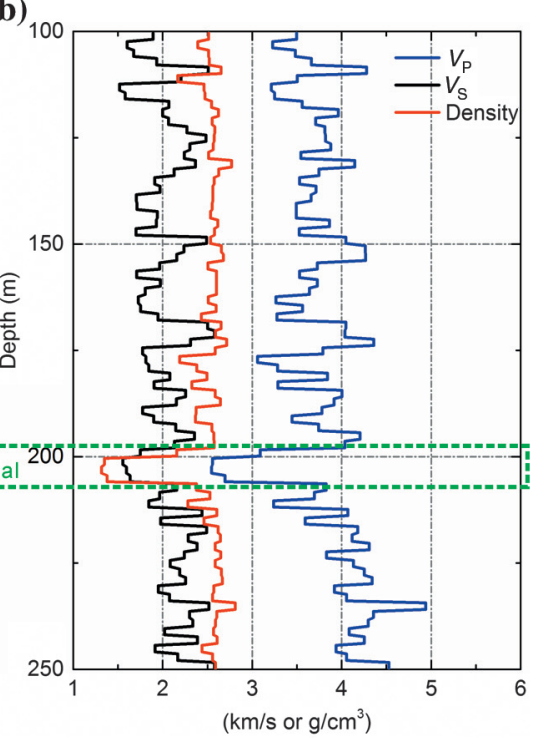

a)

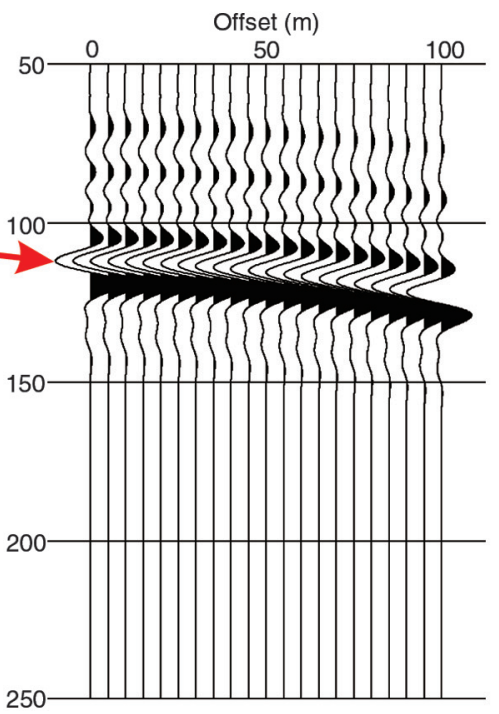

b)

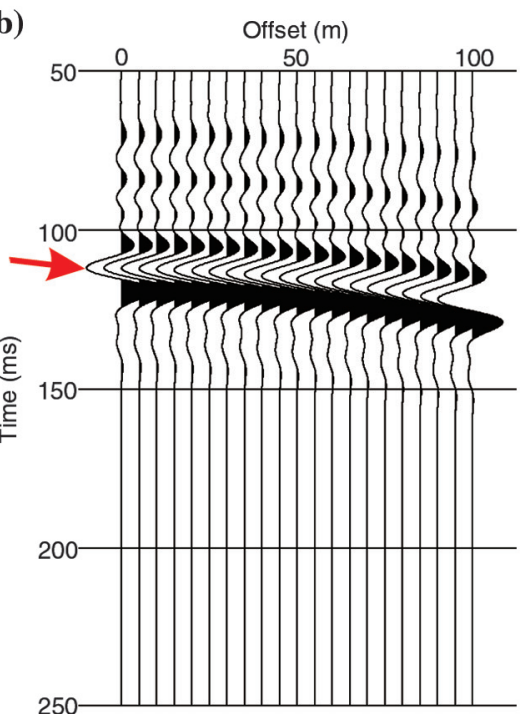

a)

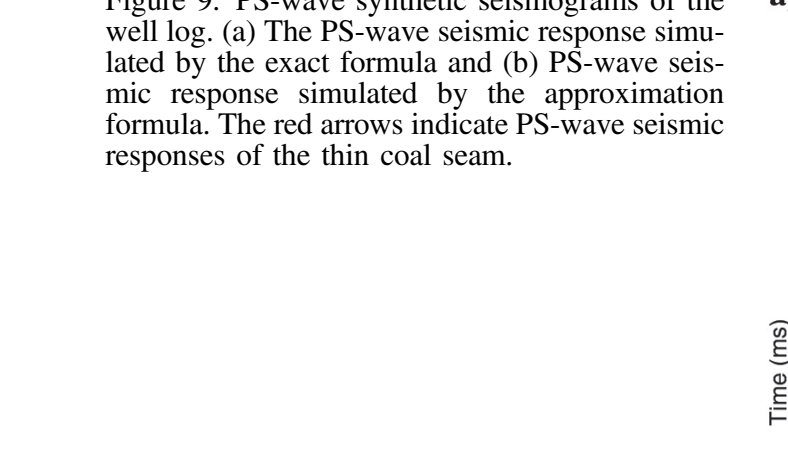
lated by the exact formula and (b) PS-wave seismic response simulated by the approximation formula. The red arrows indicate PS-wave seismic responses of the thin coal seam. b)

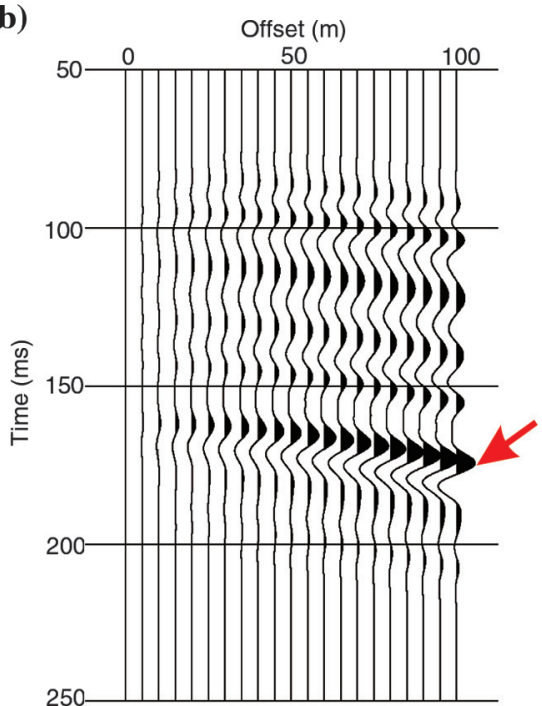


taining thin layers (Figure 7) from a coal mine in China. We compare exact and approximate reflection coefficients based synthetic seismograms.

After well-logging curves stratification, PP- and PS-waves synthetic seismograms of exact and approximate reflections are simulated and shown in Figures 8 and 9, respectively. We choose the coal seam in the well log as our investigative target, position of which is indicated by dashed green frame in Figure 7. The PP- and PS-wave seismic responses of the thin coal seam, indicated by the red arrows in synthetic seismograms, show that seismic responses simulated by the approximate reflection coefficients match very well with those simulated by the exact formula, which present quasi-Zoeppritz matrix approximation working very well in the thin-bed target.

\section{CONCLUSIONS}

This paper derives equations for thin-bed reflection/transmission coefficients defined by displacement. For the case of a single interface, the matrix equations can be simplified into the Zoeppritz equations, so that many matured AVO inversion methods for a single interface based on Zoeppritz equations and approximation formulas can be easily imitated for further thin-bed AVO inversion.

The quasi-Zoeppritz matrix approximation is established under the assumption that the middle layer is very thin. Compared with the accurate equations, the quasi-Zoeppritz matrix approximation has a compact form. The approximation reflection and transmission coefficients preserve the information of all wave modes and multiples.

The approximation errors are sensitive to the middle layer's thickness and less sensitive to incident angles except around critical angles for thicknesses smaller than $\lambda / 10$. As proved, the approximation has higher accuracy for weaker impedance difference models as well as for models with a thinner middle layer. Therefore, the assumption of a weak impedance difference may be discussed in further research.

\section{ACKNOWLEDGMENTS}

We greatly appreciate the support of the Natural Science Foundation of China (grant nos. 41425017, 41374131, and 41574126), the Open Project Program of Sinopec Key Laboratory of MultiComponent Seismic Technology ("Numerical Simulation and Analysis of Multi-Wave Seismic Data in Thin Reservoirs"), and The 13th Five-Year Plan Project Program "Seismic Wave Characteristics and Inversion of Thin Layer (2016ZX05002-005003-002)."

\section{REFERENCES}

Aki, K. I., and P. G. Richards, 1980, Quantitative seismology: W. H. Freeman \& Co.

Brekhovskikh, L. M., 1960, Waves in layered media: Academic Press

Chen, T. S., and Y. Liu, 2006, Multi-component AVO response of thin beds based on reflectance spectrum theory: Applied Geophysics, 3, 27-36, doi: 10.1007/s11770-006-0004-5.

Chung, H. M., and D. C. Lawton, 1995, Amplitude responses of thin beds: Sinusoidal approximation versus Ricker approximation: Geophysics, 60, 223-230, doi: 10.1190/1.1443750.

Kallweit, R. S., and L. C. Wood, 1982, The limits of resolution of zero-phase wavelets: Geophysics, 47, 1035-1046, doi: 10.1190/1.1441367.

Liu, Y., and D. R. Schmitt, 2003, Amplitude and AVO responses of a single thin bed: Geophysics, 68, 1161-1168, doi: 10.1190/1.1598108.

Liu, J., and K. J. Marfurt, 2006, Thin bed thickness prediction using peak instantaneous frequency: 76th Annual International Meeting, SEG, Expanded Abstracts, 968-972, doi: 10.1190/1.2370418.

Meissner, R., and E. Meixner, 1969, Deformation of seismic wavelets by thin layers and layered boundaries: Geophysical Prospecting, 17, 1-27, doi: 10.1111/j.1365-2478.1969.tb02069.x.

Pan, W., and A. Kristopher, 2013, AVO/AVF analysis of thin-bed in elastic media: 83rd Annual International Meeting, SEG, Expanded Abstracts, 373-377.

Puryear, C. I., and J. P. Castagna, 2008, Layer-thickness determination and stratigraphic interpretation using spectral inversion: Theory and application: Geophysics, 73, no. 2, R37-R48, doi: 10.1190/1.2838274.

Schoenberg, M., and J. Protazio, 1992, "Zoeppritz" rationalized and generalized to anisotropy: Journal of Seismic Exploration, 1, 125-144, doi: 10 $.1121 / 1.2029011$.

Shuey, R. T., 1985, A simplification of the Zoeppritz equations: Geophysics, 50, 609-614, doi: 10.1190/1.1442022.

Wang, Y., 1999, Approximations to the Zoeppritz equations and their use in AVO analysis: Geophysics, 64, 1920-1927, doi: 10.1190/1.1444698.

Wang, Y., J. Lu, Y. Shi, and C. Yang, 2009, PS-wave $Q$ estimation based on the P-wave $Q$ values: Journal of Geophysics and Engineering, 6, 386389, doi: $10.1088 / 1742-2132 / 6 / 4 / 006$

Widess, M. B., 1973, How thin is a thin bed?: Geophysics, 38, 1176-1180, doi: $10.1190 / 1.1440403$

Zhang, X., and X. Zheng, 2007, Thin bed identification based on attribute difference between far and near offset within prestack data: A model study: 77th Annual International Meeting, SEG, Expanded Abstracts, 293-297. 